\title{
A strengthened monotonicity inequality of quantum relative entropy: A unifying approach via Rényi relative entropy
}

\author{
Lin Zhang* \\ Institute of Mathematics, Hangzhou Dianzi University, Hangzhou 310018, PR China
}

\begin{abstract}
We derive a strengthened monotonicity inequality for quantum relative entropy by employing properties of $\alpha$-Rényi relative entropy. We develop a unifying treatment towards the improvement of some quantum entropy inequalities. In particular, an emphasis is put on a lower bound of quantum conditional mutual information (QCMI) as it gives a Pinsker-like lower bound for the QCMI. We also give some improved entropy inequalities based on Rényi relative entropy. The inequalities obtained, thus, extends some well-known ones. We also obtain a condition under which a tripartite operator becomes a Markov state. As a by-product we provide some trace inequalities of operators, which are of independent interest.
\end{abstract}

Mathematics Subject Classification. 47A63, 15A90, 46N50, 46L30, 81Q10.

Keywords. relative entropy, quantum channel, strong subadditivity, Rényi relative entropy.

\section{Introduction}

To begin with, let us fix some notations. Let $\mathcal{H}_{d}$ be a $d$-dimensional complex Hilbert space. A quantum state $\rho$ on $\mathcal{H}_{d}$ is a positive semi-definite operator of trace one and in particular, the operator $\rho=|\psi\rangle\langle\psi|$ is said to be a pure state for each unit vector $|\psi\rangle \in \mathcal{H}_{d}$. The set of all quantum states on $\mathcal{H}_{d}$ is denoted by $\mathrm{D}\left(\mathcal{H}_{d}\right)$. For each quantum state $\rho \in \mathrm{D}\left(\mathcal{H}_{d}\right)$, its von Neumann entropy is defined by $\mathrm{S}(\rho):=-\operatorname{Tr}(\rho \log \rho)$. Here and in remaining parts, all the logarithms are taken with respect to the natural base $e$. The relative entropy of two mixed states $\rho$ and $\sigma$ is defined by

$$
\mathrm{S}(\rho \| \sigma):= \begin{cases}\operatorname{Tr}(\rho(\log \rho-\log \sigma)), & \text { if } \operatorname{supp}(\rho) \subseteq \operatorname{supp}(\sigma), \\ +\infty, & \text { otherwise. }\end{cases}
$$

Here $\operatorname{supp}(\rho)(\operatorname{supp}(\sigma))$ means the support set of $\rho(\sigma)$. A quantum channel $\Phi$ over $\mathcal{H}_{d}$ is defined as a trace-preserving completely positive linear map over the set $\mathrm{D}\left(\mathcal{H}_{d}\right)$. It follows that there exist linear operators $\left\{K_{\mu}\right\}_{\mu}$ on $\mathcal{H}_{d}$ such that $\sum_{\mu} K_{\mu}^{\dagger} K_{\mu}=\mathbb{1}$ and $\Phi=\sum_{\mu} \operatorname{Ad}_{K_{\mu}}$, where $\operatorname{Ad}_{K_{\mu}}(X):=K_{\mu} X K_{\mu}^{\dagger}$, that is, for each quantum state $\rho$, we have the Kraus representation $\Phi(\rho)=\sum_{\mu} K_{\mu} \rho K_{\mu}^{+}$. A well-known property of

\footnotetext{
*E-mail: godyalin@163.com; linyz@zju.edu.cn
} 
quantum relative entropy is its monotonicity under generic quantum channels. That is,

$$
\mathrm{S}(\rho \| \sigma) \geqslant \mathrm{S}(\Phi(\rho) \| \Phi(\sigma))
$$

The condition of equality in the above equation is an interesting and important subject. An extremely important result in quantum information theory, namely, the saturation of monotonicity inequality of relative entropy under a generic quantum channel, is provided by Petz [19] and we restate it below.

Proposition 1.1 (Petz, [11, 19]). Let $\rho, \sigma \in \mathrm{D}\left(\mathcal{H}_{d}\right)$ and $\Phi$ be a quantum channel defined over $\mathcal{H}_{d}$. If $\operatorname{supp}(\rho) \subseteq$ $\operatorname{supp}(\sigma)$, then

$$
\mathrm{S}(\rho \| \sigma)=\mathrm{S}(\Phi(\rho) \| \Phi(\sigma)) \text { if and only if } \Phi_{\sigma}^{*} \circ \Phi(\rho)=\rho,
$$

where $\Phi_{\sigma}^{*}=\operatorname{Ad}_{\sigma^{1 / 2}} \circ \Phi^{*} \circ \operatorname{Ad}_{\Phi(\sigma)^{-1 / 2}}$, and $\Phi^{*}$ is the dual of $\Phi$ with respect to Hilbert-Schmidt inner product over the operator space on $\mathcal{H}_{d}$, i.e. $\operatorname{Tr}\left(\Phi^{*}(X) Y\right)=\operatorname{Tr}(X \Phi(Y))$ for all operators $X, Y$ on $\mathcal{H}_{d}$.

The well-known strong subadditivity (SSA) inequality of quantum entropy, obtained by Lieb and Ruskai in [17],

$$
\mathrm{S}\left(\rho_{A B C}\right)+\mathrm{S}\left(\rho_{B}\right) \leqslant \mathrm{S}\left(\rho_{A B}\right)+\mathrm{S}\left(\rho_{B C}\right)
$$

is a ubiquitous result in quantum information theory. It is known that SSA is equivalent to the monotonicity inequality of quantum relative entropy. Based on SSA, a new concept-conditional mutual informationis proposed by mimicking classical one. It measures the correlations of two quantum systems relative to a third: Given a tripartite state $\rho_{A B C} \in \mathrm{D}\left(\mathcal{H}_{A B C}\right)$, where $\mathcal{H}_{A B C}:=\mathcal{H}_{A} \otimes \mathcal{H}_{B} \otimes \mathcal{H}_{C}$, it is defined as

$$
I(A: C \mid B)_{\rho}:=\mathrm{S}\left(\rho_{A B}\right)+\mathrm{S}\left(\rho_{B C}\right)-\mathrm{S}\left(\rho_{A B C}\right)-\mathrm{S}\left(\rho_{B}\right) .
$$

Clearly conditional mutual information is nonnegative by SSA. Thus, getting a lower bound on conditional mutual information is equivalent to the tightening of SSA and is an important line of research. Hence, characterization of vanishing conditional mutual information is a first step to this problem.

Ruskai is the first one to discuss the equality condition of SSA, i.e. vanishing conditional mutual information. By analyzing the equality condition of Golden-Thompson inequality, she obtained the following characterization [21]:

$$
I(A: C \mid B)_{\rho}=0 \Longleftrightarrow \log \rho_{A B C}+\log \rho_{B}=\log \rho_{A B}+\log \rho_{B C}
$$

Throughout the present paper, we have suppressed implicit tensor products with the identity by conventions. For example, $\log \rho_{A B}$ means $\left(\log \rho_{A B}\right) \otimes \mathbb{1}_{C}$, where $\rho_{A B}=\operatorname{Tr}_{C}\left(\rho_{A B C}\right)$ is the reduced state (or density operator) of the system $A B$.

Later on, using the relative modular approach established by Araki, Petz gave another characterization of the equality condition of SSA [20]:

$$
I(A: C \mid B)_{\rho}=0 \Longleftrightarrow \rho_{A B C}^{\mathrm{i} t} \rho_{B C}^{-\mathrm{i} t}=\rho_{A B}^{\mathrm{i} t} \rho_{B}^{-\mathrm{i} t} \quad(\forall t \in \mathbb{R}),
$$

where $\mathrm{i}=\sqrt{-1}$ is the imaginary unit. From this, we see that $I(A: C \mid B)_{\rho}>0$ if and only if $\rho_{A B C}^{\mathrm{it}} \neq$ $\rho_{A B}^{\mathrm{i} t} \rho_{B}^{-\mathrm{i} t} \rho_{B C}^{\mathrm{i} t}$ for all $t \in \mathbb{R}$. Therefore, comparing both $I(A: C \mid B)_{\rho}$ and $\left\|\rho_{A B C}^{\mathrm{i} t}-\rho_{A B}^{\mathrm{i} t} \rho_{B}^{-\mathrm{i} t} \rho_{B C}^{\mathrm{i} t}\right\|$, where $\|*\|$ 
is a metric over the unitary group, is mathematically interesting. For instance, they have a property in common: If $\rho, \sigma \in \mathrm{D}\left(\mathcal{H}_{d}\right)$, then $\|\rho-\sigma\|_{1} \leqslant 2$ and $\left\|\rho^{\mathrm{i} t}-\sigma^{\mathrm{i} t}\right\|_{\infty} \leqslant 2$, where $\|*\|_{\infty}$ is the spectral norm of an operator.

Hayden et al. in [10] have shown that $I(A: C \mid B)_{\rho}=0$ if and only if the following conditions hold:

(i) $\mathcal{H}_{B}=\bigoplus_{k} \mathcal{H}_{b_{k}^{L}} \otimes \mathcal{H}_{b_{k}^{R}}$

(ii) $\rho_{A B C}=\bigoplus_{k} p_{k} \rho_{A b_{k}^{L}} \otimes \rho_{b_{k}^{R} C}$, where $\rho_{A b_{k}^{L}} \in \mathrm{D}\left(\mathcal{H}_{A} \otimes \mathcal{H}_{b_{k}^{L}}\right), \rho_{b_{k}^{R} C} \in \mathrm{D}\left(\mathcal{H}_{b_{k}^{R}} \otimes \mathcal{H}_{C}\right)$ for each index $k$; and $\left\{p_{k}\right\}$ is a probability distribution.

In order to get rid of the above-known difficult computation such as logarithm and complex exponential power of states, Zhang [25] gave another new characterization of vanishing conditional mutual information. Specifically, define

$$
M:=\left(\rho_{A B}^{1 / 2} \otimes \mathbb{1}_{C}\right)\left(\mathbb{1}_{A} \otimes \rho_{B}^{-1 / 2} \otimes \mathbb{1}_{C}\right)\left(\mathbb{1}_{A} \otimes \rho_{B C}^{1 / 2}\right) \equiv \rho_{A B}^{1 / 2} \rho_{B}^{-1 / 2} \rho_{B C}^{1 / 2} .
$$

Then the following conditions are equivalent:

(i) The vanishing of conditional mutual information, i.e. $I(A: C \mid B)_{\rho}=0$.

(ii) $\rho_{A B C}=M M^{\dagger}=\rho_{A B}^{1 / 2} \rho_{B}^{-1 / 2} \rho_{B C} \rho_{B}^{-1 / 2} \rho_{A B}^{1 / 2}$.

(iii) $\rho_{A B C}=M^{\dagger} M=\rho_{B C}^{1 / 2} \rho_{B}^{-1 / 2} \rho_{A B} \rho_{B}^{-1 / 2} \rho_{B C}^{1 / 2}$.

With these characterizations of vanishing conditional mutual information, one starts to make an attempt to get a lower bound on the QCMI. In [4], Brandão et al. first obtained the following lower bound for $I(A: C \mid B)_{\rho}$ :

$$
I(A: C \mid B)_{\rho} \geqslant \frac{1}{8} \min _{\sigma_{A C} \in \mathbb{S E P}}\left\|\rho_{A C}-\sigma_{A C}\right\|_{1-\mathbb{L O C C}}^{2}
$$

where

$$
\left\|\rho_{A C}-\sigma_{A C}\right\|_{1-\mathbb{L O C C}}^{2} \stackrel{\text { def }}{=} \sup _{\mathcal{M} \in 1-\mathbb{L O C C}}\left\|\mathcal{M}\left(\rho_{A C}\right)-\mathcal{M}\left(\sigma_{A C}\right)\right\|_{1} .
$$

Here $\operatorname{SEP}$ means the set of all separable states over the bipartite cut $A: C$; 1 -LOCC means the oneway LOCC measurement. They used many advanced mathematical techniques to get this result in their paper. Based on this result, they cracked a long-standing open problem in quantum information theory: the squashed entanglement is faithful. Later, $\mathrm{Li}$ and Winter in [14] gave another approach to study the same problem and have improved the lower bound for $I(A: C \mid B)_{\rho}$ :

$$
I(A: C \mid B)_{\rho} \geqslant \frac{1}{2} \min _{\sigma_{A C} \in \operatorname{SEP}}\left\|\rho_{A C}-\sigma_{A C}\right\|_{1-\mathbb{L O C C}}^{2} .
$$

A different approach is taken by Ibinson et al. in [13]. They studied the robustness of quantum Markov chains, i.e. the perturbation to the states with vanishing conditional mutual information. They found that the quantum Markov chains are not robust because, even if the conditional mutual information is small, the original tripartite state can deviate a lot from Markov chains.

Several breakthroughs about the investigation of bounding the small conditional mutual information are made, respectively, by Fawzi and Renner [9], Wilde et. al [2, 3, 8, 23], Brandão [5], Li and Winter [14, 15], 
and, Zhang and $\mathrm{Wu}$ [27]. In this paper, we give a unifying treatment for some entropy inequalities and improvement of monotonicity inequality of relative entropy under unital quantum channels by employing quantum $\alpha$-Rényi relative entropy [18]. Once we get one of our main results (Theorem [3.8), we can simply derive all improved versions of some quantum entropy inequalities [7]. Note that our method is different from that in [7], and is much simpler compared to the one in [27].

The paper is organized as follows. The definition and properties of quantum Rényi relative entropy are given in Section 2 Section 3 deals with the main results and their consequences. In the Section 4. we summarize a series of strengthened entropy inequalities. The discussion and concluding remarks are presented in Section 5 Some questions are left open for the future research.

\section{Quantum $\alpha$-Rényi relative entropy}

The quantum $\alpha$-Rényi relative entropy is defined as follows [18]:

$$
\mathrm{S}_{\alpha}(\rho \| \sigma):=\frac{1}{\alpha-1} \log \operatorname{Tr}\left(\rho^{\alpha} \sigma^{1-\alpha}\right),
$$

where $\rho, \sigma \in \mathrm{D}\left(\mathcal{H}_{d}\right)$, and a parameter $\alpha \in(0,1)$. Two important properties of $\alpha$-Rényi relative entropy used in this paper are listed below: it holds that

(i) $\mathrm{S}(\rho \| \sigma)=\lim _{\alpha \rightarrow 1^{-}} \mathrm{S}_{\alpha}(\rho \| \sigma)$, thus we denote $\mathrm{S}(\rho \| \sigma)=\mathrm{S}_{1^{-}}(\rho \| \sigma)$;

(ii) $\alpha \mapsto \mathrm{S}_{\alpha}(\rho \| \sigma)$ is monotonically increasing on $(0,1)$.

Hence, if $\alpha \geqslant \frac{1}{2}$, then $\mathrm{S}_{1 / 2}(\rho \| \sigma) \leqslant \mathrm{S}_{\alpha}(\rho \| \sigma)$. Taking the limit for $\alpha \rightarrow 1^{-}$on the right hand side of the inequality, we have the following important result 1 :

$$
\mathrm{S}(\rho \| \sigma) \geqslant-2 \log \operatorname{Tr}(\sqrt{\rho} \sqrt{\sigma})
$$

for two states $\rho$ and $\sigma$. The same inequality is obtained by Carlen and Lieb [7] using Peierls-Bogoliubov inequality and Golden-Thompson inequality. Later, in our further investigations, we find that this inequality seems to improve some of the entropy inequalities obtained recently. Compared with Pinsker's bound for relative entropy

$$
\mathrm{S}(\rho \| \sigma) \geqslant \frac{1}{2}\|\rho-\sigma\|_{1}^{2},
$$

the lower bound in Eq. (2.2) for the relative entropy is very useful in our present paper. Note that there is an identity which will be used in our treatment:

$$
\mathrm{S}(\rho \| \mu \sigma)=\mathrm{S}(\rho \| \sigma)-\log \mu, \quad \forall \mu>0 .
$$

\section{Main results}

In this section, we prove our main theorem. We take a unifying method to treat improvement of some entropy inequalities. The proof strategy followed here is completely different from that used by Carlen

\footnotetext{
${ }^{1}$ This method to get the inequality is pointed out to the author by M. Wilde.
} 
and Lieb [7]. In the following proposition, we present a very simple and natural proof following our unifying approach.

Proposition 3.1. For two states $\rho, \sigma \in \mathrm{D}\left(\mathcal{H}_{d}\right)$ and a quantum channel $\Phi$ over $\mathcal{H}_{d}$, we have

$$
\begin{aligned}
& \mathrm{S}(\rho \| \sigma)-\mathrm{S}(\Phi(\rho) \| \Phi(\sigma)) \\
& \geqslant-2 \log \operatorname{Tr}\left(\sqrt{\rho} \sqrt{\exp \left[\log \sigma+\Phi^{*}(\log \Phi(\rho))-\Phi^{*}(\log \Phi(\sigma))\right]}\right) .
\end{aligned}
$$

Proof. Define a state as follows:

$$
\omega=\lambda^{-1} \exp \left(\log \sigma+\Phi^{*}(\log \Phi(\rho)-\log \Phi(\sigma))\right),
$$

with $\lambda:=\operatorname{Tr}\left(\exp \left(\log \sigma+\Phi^{*}(\log \Phi(\rho)-\log \Phi(\sigma))\right)\right)>0$. Multiplying by $\lambda$ on both sides of above equation and then taking logarithm, we get

$$
\log \sigma+\Phi^{*}(\log \Phi(\rho)-\log \Phi(\sigma))=\log (\lambda \omega) .
$$

Now consider

$$
\begin{aligned}
& \mathrm{S}(\rho \| \sigma)-\mathrm{S}(\Phi(\rho) \| \Phi(\sigma)) \\
& =\operatorname{Tr}(\rho(\log \rho-\log \sigma))-\operatorname{Tr}(\Phi(\rho)(\log \Phi(\rho)-\log \Phi(\sigma))) \\
& =\operatorname{Tr}(\rho(\log \rho-\log \sigma))-\operatorname{Tr}\left(\rho \Phi^{*}(\log \Phi(\rho)-\log \Phi(\sigma))\right) \\
& =\operatorname{Tr}\left(\rho\left(\log \rho-\left[\log \sigma+\Phi^{*}(\log \Phi(\rho)-\log \Phi(\sigma))\right]\right)\right) .
\end{aligned}
$$

Using Eq. (3.1), we have

$$
\begin{aligned}
\mathrm{S}(\rho \| \sigma)-\mathrm{S}(\Phi(\rho) \| \Phi(\sigma)) & =\mathrm{S}(\rho \| \lambda \omega) \\
& =\mathrm{S}(\rho \| \omega)-\log \lambda \\
& \geqslant-2 \log \operatorname{Tr}(\sqrt{\rho} \sqrt{\omega})-\log \lambda
\end{aligned}
$$

Since

$$
\sqrt{\omega}=\lambda^{-1 / 2} \sqrt{\exp \left(\log \sigma+\Phi^{*}(\log \Phi(\rho)-\log \Phi(\sigma))\right)},
$$

it follows that

$$
-2 \log \operatorname{Tr}(\sqrt{\rho} \sqrt{\omega})-\log \lambda=-2 \log \operatorname{Tr}\left(\sqrt{\rho} \sqrt{\exp \left(\log \sigma+\Phi^{*}(\log \Phi(\rho)-\log \Phi(\sigma))\right)}\right) .
$$

This completes the proof.

Remark 3.2. For any given two positive semi-definite matrices $M$ and $N$, it holds [27] that

$$
\|\sqrt{M}-\sqrt{N}\|_{2}^{2} \leqslant\|M-N\|_{1} \leqslant\|\sqrt{M}-\sqrt{N}\|_{2}\|\sqrt{M}+\sqrt{N}\|_{2}^{\prime}
$$

where $\|X\|_{p}:=\left(\operatorname{Tr}\left(|X|^{p}\right)\right)^{1 / p}$ is Schatten $p$-norm for positive integers $p$, and $|X|=\sqrt{X^{\dagger} X}$. Indeed, the proof of Eq. (3.2) uses the well-known inequality in matrix analysis, i.e. Audenaert's inequality [1]:

$$
\operatorname{Tr}\left(M^{t} N^{1-t}\right) \geqslant \frac{1}{2} \operatorname{Tr}(M+N-|M-N|)
$$


for all $t \in[0,1]$ and positive matrices $M, N$. If both the traces of $M$ and $N$ are no more than one, i.e. $\operatorname{Tr}(M), \operatorname{Tr}(N) \leqslant 1$, then we see from the proof of [27, Theorem 2.1] that

$$
\operatorname{Tr}(\sqrt{M} \sqrt{N}) \leqslant 1-\frac{1}{2}\|\sqrt{M}-\sqrt{N}\|_{2}^{2} .
$$

Furthermore,

$$
-2 \log \operatorname{Tr}(\sqrt{M} \sqrt{N}) \geqslant-2 \log \left(1-\frac{1}{2}\|\sqrt{M}-\sqrt{N}\|_{2}^{2}\right) \geqslant\|\sqrt{M}-\sqrt{N}\|_{2}^{2},
$$

where we used the fact that $-\log (1-t) \geqslant t$ for $t \leqslant 1$.

Proposition 3.3 (Lieb, [16]). For a fixed Hermitian matrix $H \in M_{d}(\mathbb{C})$, the following map

$$
X \mapsto \operatorname{Tr}\left(e^{H+\log X}\right)
$$

is concave over the set $\mathrm{Pd}\left(\mathbb{C}^{d}\right)$ of all positive definite matrices of order $d$.

Proposition 3.4. For two arbitrary bipartite states $\rho_{A B}, \sigma_{A B} \in \mathrm{D}\left(\mathcal{H}_{A B}\right)$ with $\mathcal{H}_{A B}=\mathcal{H}_{A} \otimes \mathcal{H}_{B}$, it holds that

$$
\begin{aligned}
\mathrm{S}\left(\rho_{A B} \| \sigma_{A B}\right)-\mathrm{S}\left(\rho_{A} \| \sigma_{A}\right) & \geqslant-2 \log \operatorname{Tr}\left(\sqrt{\rho_{A B}} \sqrt{\exp \left(\log \sigma_{A B}-\log \sigma_{A}+\log \rho_{A}\right)}\right) \\
& \geqslant\left\|\sqrt{\rho_{A B}}-\sqrt{\exp \left(\log \sigma_{A B}-\log \sigma_{A}+\log \rho_{A}\right)}\right\|_{2}^{2} .
\end{aligned}
$$

In particular, $\mathrm{S}\left(\rho_{A B} \| \sigma_{A B}\right)=\mathrm{S}\left(\rho_{A} \| \sigma_{A}\right)$ if and only if $\log \rho_{A B}-\log \rho_{A}=\log \sigma_{A B}-\log \sigma_{A}$.

Proof. In Proposition 3.1, letting $\rho=\rho_{A B}, \sigma=\sigma_{A B}$, and the quantum channel $\Phi=\operatorname{Tr}_{B}$ (a partial trace over system $B$ ), we obtain the first inequality. The second inequality follows from Remark 3.2 due to the fact that $\operatorname{Tr}\left(\exp \left(\log \sigma_{A B}-\log \sigma_{A}+\log \rho_{A}\right)\right) \leqslant 1$. Indeed, let $H=\log \rho_{A}-\log \sigma_{A}$ and $X=\sigma_{A B}$ in Proposition 3.3, it follows that

$$
\begin{aligned}
& \operatorname{Tr}\left(\exp \left(\log \rho_{A}-\log \sigma_{A}+\log \sigma_{A B}\right)\right) \\
& =\int_{\mathrm{U}\left(d_{B}\right)} \operatorname{Tr}\left(\exp \left(\log \rho_{A} \otimes \mathbb{1}_{B}-\log \sigma_{A} \otimes \mathbb{1}_{B}+\log \left(U_{B} \sigma_{A B} U_{B}^{\dagger}\right)\right)\right) d U_{B} \\
& \leqslant \operatorname{Tr}\left(\exp \left(\log \rho_{A} \otimes \mathbb{1}_{B}-\log \sigma_{A} \otimes \mathbb{1}_{B}+\log \left[\int_{\mathrm{U}\left(d_{B}\right)} U_{B} \sigma_{A B} U_{B}^{\dagger} \mathrm{d} U_{B}\right]\right)\right) \\
& =\operatorname{Tr}\left(\exp \left(\log \rho_{A} \otimes \mathbb{1}_{B}-\log \sigma_{A} \otimes \mathbb{1}_{B}+\log \left[\sigma_{A} \otimes \mathbb{1}_{B} / d_{B}\right]\right)\right) \\
& =\operatorname{Tr}\left(\exp \left(\log \rho_{A} \otimes \mathbb{1}_{B}-\log \sigma_{A} \otimes \mathbb{1}_{B}+\log \sigma_{A} \otimes \mathbb{1}_{B}+\mathbb{1}_{A} \otimes \log \left(\mathbb{1}_{B} / d_{B}\right)\right)\right) \\
& =\operatorname{Tr}\left(\rho_{A} \otimes \mathbb{1}_{B}\right) / d_{B}=1 .
\end{aligned}
$$

This concludes the proof.

The above inequality is firstly derived by Carlen and Lieb as one of their main results in [7]. The following result is a direct consequence of it. In addition, we present here another approach to get it.

Corollary 3.5. For an arbitrary tripartite state $\rho_{A B C}$, we have that

$$
\begin{aligned}
I(A: C \mid B)_{\rho} & \geqslant-2 \log \operatorname{Tr}\left(\sqrt{\rho_{A B C}} \sqrt{\exp \left(\log \rho_{A B}-\log \rho_{B}+\log \rho_{B C}\right)}\right) \\
& \geqslant\left\|\sqrt{\rho_{A B C}}-\sqrt{\exp \left(\log \rho_{A B}-\log \rho_{B}+\log \rho_{B C}\right)}\right\|_{2}^{2} .
\end{aligned}
$$

In particular, the conditional mutual information vanishes if and only if $\log \rho_{A B C}+\log \rho_{B}=\log \rho_{A B}+\log \rho_{B C}$. 
Proof. Note that the quantum conditional mutual information $I(A: C \mid B)_{\rho}$ can be rewritten as follows:

$$
I(A: C \mid B)_{\rho}=\mathrm{S}\left(\rho_{A B C} \| \omega_{A B C}\right)-\log \lambda
$$

where $\lambda \omega_{A B C}=\exp \left(\log \rho_{A B}-\log \rho_{B}+\log \rho_{B C}\right)$ and $\lambda=\operatorname{Tr}\left(\exp \left(\log \rho_{A B}-\log \rho_{B}+\log \rho_{B C}\right)\right)$. By using (2.2), we get

$$
\begin{aligned}
I(A: C \mid B)_{\rho} & \geqslant-2 \log \operatorname{Tr}\left(\sqrt{\rho_{A B C}} \sqrt{\omega_{A B C}}\right)-\log \lambda \\
& =-2 \log \operatorname{Tr}\left(\sqrt{\rho_{A B C}} \sqrt{\exp \left(\log \rho_{A B}-\log \rho_{B}+\log \rho_{B C}\right)}\right) .
\end{aligned}
$$

This is the first inequality. The second approach to the first inequality is by using Theorem 3.8. By letting $\Phi=\operatorname{Tr}_{A}, \rho=\rho_{A B C}$ and $\sigma=\rho_{A B} \otimes \rho_{C}$, it follows that

$$
I(A: C \mid B)_{\rho}=\mathrm{S}(\rho \| \sigma)-\mathrm{S}(\Phi(\rho) \| \Phi(\sigma)) .
$$

By employing Proposition 3.1, we have

$$
I(A: C \mid B)_{\rho} \geqslant-2 \log \operatorname{Tr}\left(\sqrt{\rho_{A B C}} \sqrt{\exp \left(\log \rho_{A B}-\log \rho_{B}+\log \rho_{B C}\right)}\right) .
$$

The second inequality follows directly from Remark 3.2 due to the fact [21] that

$$
\operatorname{Tr}\left(\exp \left(\log \rho_{A B}-\log \rho_{B}+\log \rho_{B C}\right)\right) \leqslant 1 .
$$

Here we give another proof of this inequality. Indeed, let $H=\log \rho_{A B}-\log \rho_{B}$ and $X=\rho_{B C}$ in Proposition 3.3 , it follows that

$$
\begin{aligned}
& \operatorname{Tr}\left(\exp \left(\log \rho_{A B}-\log \rho_{B}+\log \rho_{B C}\right)\right) \\
& =\int_{\mathrm{U}\left(d_{C}\right)} \operatorname{Tr}\left(\exp \left(\log \rho_{A B} \otimes \mathbb{1}_{C}-\mathbb{1}_{A} \otimes \log \rho_{B} \otimes \mathbb{1}_{C}+\mathbb{1}_{A} \otimes \log \left(U_{C} \rho_{B C} U_{C}^{+}\right)\right)\right) \mathrm{d} U_{C} \\
& \leqslant \operatorname{Tr}\left(\exp \left(\log \rho_{A B} \otimes \mathbb{1}_{C}-\mathbb{1}_{A} \otimes \log \rho_{B} \otimes \mathbb{1}_{C}+\mathbb{1}_{A} \otimes \log \left[\int_{\mathrm{U}\left(d_{C}\right)} U_{C} \rho_{B C} U_{C}^{+} \mathrm{d} U_{C}\right]\right)\right) \\
& =\operatorname{Tr}\left(\exp \left(\log \rho_{A B} \otimes \mathbb{1}_{C}-\mathbb{1}_{A} \otimes \log \rho_{B} \otimes \mathbb{1}_{C}+\mathbb{1}_{A} \otimes \log \left[\rho_{B} \otimes \mathbb{1}_{C} / d_{C}\right]\right)\right) \\
& =\operatorname{Tr}\left(\exp \left(\log \rho_{A B} \otimes \mathbb{1}_{C}-\mathbb{1}_{A} \otimes \log \rho_{B} \otimes \mathbb{1}_{C}+\mathbb{1}_{A} \otimes \log \rho_{B} \otimes \mathbb{1}_{C}+\mathbb{1}_{A} \otimes \mathbb{1}_{B} \otimes \log \left(\mathbb{1}_{C} / d_{C}\right)\right)\right) \\
& =\operatorname{Tr}\left(\rho_{A B} \otimes \mathbb{1}_{C}\right) / d_{C}=1 .
\end{aligned}
$$

Now if the conditional mutual information vanishes, then

$$
\left\|\sqrt{\rho_{A B C}}-\sqrt{\exp \left(\log \rho_{A B}+\log \rho_{B C}-\log \rho_{B}\right)}\right\|_{2}=0,
$$

that is, $\sqrt{\rho_{A B C}}=\sqrt{\exp \left(\log \rho_{A B}+\log \rho_{B C}-\log \rho_{B}\right)}$, which is equivalent to the following:

$$
\rho_{A B C}=\exp \left(\log \rho_{A B}+\log \rho_{B C}-\log \rho_{B}\right) .
$$

By taking logarithm over both sides, it is seen that $\log \rho_{A B C}=\log \rho_{A B}+\log \rho_{B C}-\log \rho_{B}$, a well-known equality condition of strong subadditivity obtained by Ruskai in [21]. This completes the proof. 
Remark 3.6. Note that our technique used in the proof of the inequality (3.10) implies a more general result: if $\rho_{A B C}, \sigma_{A B C}, \tau_{A B C}$ are tripartite states on $\mathcal{H}_{A B C}$ satisfying the condition that $\rho_{B}=\sigma_{B}$ or $\sigma_{B}=\tau_{B}$, then

$$
\operatorname{Tr}\left(\exp \left(\log \rho_{A B}-\log \sigma_{B}+\log \tau_{B C}\right)\right) \leqslant 1 .
$$

Now we will give an important lemma. In fact, it is based on a famous Lieb's concavity result, i.e. Proposition 3.3 .

Lemma 3.7. For given two states $\rho, \sigma \in \mathrm{D}\left(\mathcal{H}_{d}\right)$ and a unital quantum channel $\Phi$ defined over $\mathcal{H}_{d}$, i.e. $\Phi\left(\mathbb{1}_{d}\right)=\mathbb{1}_{d}$ and $\Phi^{*}\left(\mathbb{1}_{d}\right)=\mathbb{1}_{d}$, we have

$$
\operatorname{Tr}\left(\exp \left(\log \sigma+\Phi^{*}(\log \Phi(\rho))-\Phi^{*}(\log \Phi(\sigma))\right)\right) \leqslant 1
$$

Proof. By Stinespring's dilation theorem, a given quantum channel $\Phi$ can be realized as

$$
\Phi(X)=\operatorname{Tr}_{B}\left(U\left(X \otimes \widehat{\mathbb{1}}_{B}\right) U^{+}\right)
$$

for some unitary $U$ and completely mixed state $\widehat{\mathbb{1}}_{B}:=\mathbb{1}_{B} / d_{B}$ in an auxiliary Hilbert space $\mathcal{H}_{B}$. The dual of $\Phi$ is given by $\Phi^{*}(Y)=\operatorname{Tr}_{B}\left(U^{\dagger}\left(Y \otimes \widehat{\mathbb{1}}_{B}\right) U\right)$. Denote $\rho_{A B}:=U\left(\rho \otimes \widehat{\mathbb{1}}_{B}\right) U^{\dagger}$ and $\sigma_{A B}:=U\left(\sigma \otimes \widehat{\mathbb{1}}_{B}\right) U^{\dagger}$. Then $\rho_{A}=\operatorname{Tr}_{B}\left(\rho_{A B}\right)=\Phi(\rho)$ and $\sigma_{A}=\operatorname{Tr}_{B}\left(\sigma_{A B}\right)=\Phi(\sigma)$. Thus by the techniques in the proof of Proposition 3.4,

$$
\operatorname{Tr}\left(\exp \left(\log \rho_{A} \otimes \mathbb{1}_{B}-\log \sigma_{A} \otimes \mathbb{1}_{B}+\log \sigma_{A B}\right)\right) \leqslant 1 .
$$

That is,

$$
\begin{aligned}
1 & \geqslant \operatorname{Tr}\left(\exp \left(\log \Phi(\rho) \otimes \mathbb{1}_{B}-\log \Phi(\sigma) \otimes \mathbb{1}_{B}+\log \left[U\left(\sigma \otimes \widehat{\mathbb{1}}_{B}\right) U^{\dagger}\right]\right)\right) \\
& =\operatorname{Tr}\left(\exp \left(U^{\dagger}\left[\log \Phi(\rho) \otimes \mathbb{1}_{B}-\log \Phi(\sigma) \otimes \mathbb{1}_{B}\right] U+\log \left(\sigma \otimes \widehat{\mathbb{1}}_{B}\right)\right)\right) \\
& =\int_{\mathrm{U}\left(d_{B}\right)} \operatorname{Tr}\left(\exp \left(U^{\dagger}\left[\log \Phi(\rho) \otimes \mathbb{1}_{B}-\log \Phi(\sigma) \otimes \mathbb{1}_{B}\right] U+\log \left(\sigma \otimes \widehat{\mathbb{1}}_{B}\right)\right)\right) \mathrm{d} V_{B} \\
& =\int_{\mathrm{U}\left(d_{B}\right)} \operatorname{Tr}\left(\exp \left(\left(\mathbb{1} \otimes V_{B}\right) U^{\dagger}\left[\log \Phi(\rho) \otimes \mathbb{1}_{B}-\log \Phi(\sigma) \otimes \mathbb{1}_{B}\right] U\left(\mathbb{1} \otimes V_{B}\right)^{\dagger}+\log \left(\sigma \otimes \widehat{\mathbb{1}}_{B}\right)\right)\right) \mathrm{d} V_{B} \\
& \geqslant \operatorname{Tr}\left(\exp \left(\int_{\mathrm{U}\left(d_{B}\right)}\left(\mathbb{1} \otimes V_{B}\right) U^{\dagger}\left[\log \Phi(\rho) \otimes \mathbb{1}_{B}-\log \Phi(\sigma) \otimes \mathbb{1}_{B}\right] U\left(\mathbb{1} \otimes V_{B}\right)^{\dagger} \mathrm{d} V_{B}+\log \left(\sigma \otimes \widehat{\mathbb{1}}_{B}\right)\right)\right) \\
& =\operatorname{Tr}\left(\exp \left(\operatorname{Tr}_{B}\left(U^{\dagger}\left[(\log \Phi(\rho)-\log \Phi(\sigma)) \otimes \mathbb{1}_{B}\right] U\right) \otimes \widehat{\mathbb{1}}_{B}+\log \left(\sigma \otimes \widehat{\mathbb{1}}_{B}\right)\right)\right) \\
& =\operatorname{Tr}\left(\exp \left(\operatorname{Tr}_{B}\left(U^{\dagger}\left[(\log \Phi(\rho)-\log \Phi(\sigma)) \otimes \widehat{\mathbb{1}}_{B}\right] U\right) \otimes \mathbb{1}_{B}+\log \left(\sigma \otimes \widehat{\mathbb{1}}_{B}\right)\right)\right),
\end{aligned}
$$

where we used the following facts: $X \mapsto \operatorname{Tr}(\exp (X))$ is a convex functional over $\operatorname{Pd}\left(\mathbb{C}^{n}\right)$ and [26]:

$$
\int_{\mathrm{U}\left(d_{B}\right)}\left(\mathbb{1} \otimes V_{B}\right)\left[U\left(X \otimes \widehat{\mathbb{1}}_{B}\right) U^{\dagger}\right]\left(\mathbb{1} \otimes V_{B}\right)^{\dagger} \mathrm{d} V_{B}=\Phi(X) \otimes \widehat{\mathbb{1}}_{B}
$$


This indicates that

$$
\begin{aligned}
1 & \geqslant \operatorname{Tr}\left(\exp \left(\Phi^{*}(\log \Phi(\rho)-\log \Phi(\sigma)) \otimes \mathbb{1}_{B}+\log \left(\sigma \otimes \widehat{\mathbb{1}}_{B}\right)\right)\right) \\
& =\operatorname{Tr}\left(\exp \left(\Phi^{*}(\log \Phi(\rho)-\log \Phi(\sigma)) \otimes \mathbb{1}_{B}+\log \sigma \otimes \mathbb{1}_{B}+\mathbb{1}_{A} \otimes \log \left(\widehat{\mathbb{1}}_{B}\right)\right)\right) \\
& =\operatorname{Tr}\left(\exp \left(\left[\Phi^{*}(\log \Phi(\rho)-\log \Phi(\sigma))+\log \sigma\right] \otimes \mathbb{1}_{B}+\mathbb{1}_{A} \otimes \log \left(\widehat{\mathbb{1}}_{B}\right)\right)\right) \\
& =\operatorname{Tr}\left(\exp \left(\left[\Phi^{*}(\log \Phi(\rho)-\log \Phi(\sigma))+\log \sigma\right]\right) \otimes \exp \left(\log \left(\widehat{\mathbb{1}}_{B}\right)\right)\right) \\
& =\operatorname{Tr}\left(\exp \left(\left[\Phi^{*}(\log \Phi(\rho)-\log \Phi(\sigma))+\log \sigma\right]\right)\right) \operatorname{Tr}\left(\widehat{\mathbb{1}}_{B}\right) \\
& =\operatorname{Tr}\left(\exp \left(\left[\Phi^{*}(\log \Phi(\rho)-\log \Phi(\sigma))+\log \sigma\right]\right)\right),
\end{aligned}
$$

where we used the fact that $\exp (X \otimes \mathbb{1}+\mathbb{1} \otimes Y)=\exp (X) \otimes \exp (Y)$ for positive definite operators $X$ and $Y$.

Note that Eq. (3.12) is also obtained very recently by Datta and Wilde [8]. The approach used here is very simple and completely different from the one used by them. Now, we may present our main result - the strengthened monotonicity inequality of relative entropy-which is described as follows:

Theorem 3.8. For any states $\rho, \sigma \in \mathrm{D}\left(\mathcal{H}_{d}\right)$, and $\Phi$ a unital quantum channel over $\mathcal{H}_{d}$, we have

$$
\mathrm{S}(\rho \| \sigma)-\mathrm{S}(\Phi(\rho) \| \Phi(\sigma)) \geqslant\left\|\sqrt{\rho}-\sqrt{\exp \left(\log \sigma+\Phi^{*}(\log \Phi(\rho))-\Phi^{*}(\log \Phi(\sigma))\right)}\right\|_{2}^{2} .
$$

Proof. The proof follows immediately from Lemma 3.7 and Remark 3.2

Corollary 3.9. With the above notations, we have the following inequalities:

(i) Strengthened monotonicity inequality of relative entropy under a unital quantum channel:

$$
\mathrm{S}(\rho \| \sigma)-\mathrm{S}(\Phi(\rho) \| \Phi(\sigma)) \geqslant \frac{1}{4}\left\|\rho-\exp \left(\log \sigma+\Phi^{*}(\log \Phi(\rho))-\Phi^{*}(\log \Phi(\sigma))\right)\right\|_{1}^{2}
$$

(ii) Strengthened monotonicity inequality of relative entropy under partial trace:

$$
\mathrm{S}\left(\rho_{A B} \| \sigma_{A B}\right)-\mathrm{S}\left(\rho_{A} \| \sigma_{A}\right) \geqslant \frac{1}{4}\left\|\rho_{A B}-\exp \left(\log \sigma_{A B}-\log \sigma_{A}+\log \rho_{A}\right)\right\|_{1}^{2} .
$$

(iii) Strengthened subadditivity inequality of quantum entropy:

$$
I(A: C \mid B)_{\rho} \geqslant \frac{1}{4}\left\|\rho_{A B C}-\exp \left(\log \rho_{A B}+\log \rho_{B C}-\log \rho_{B}\right)\right\|_{1}^{2} .
$$

\section{On some entropy inequalities}

From our results from the previous sections and the following result, we can derive many strengthened entropy inequalities.

Proposition 4.1. For a state $\rho \in \mathrm{D}\left(\mathcal{H}_{d}\right)$ and a subnormalized state $\sigma$ on $\mathcal{H}_{d}$ (i.e. $\operatorname{Tr}(\sigma) \leqslant 1$ ), it holds that

$$
\begin{aligned}
\mathrm{S}(\rho \| \sigma) & \geqslant-2 \log \operatorname{Tr}(\sqrt{\rho} \sqrt{\sigma}) \\
& \geqslant\|\sqrt{\rho}-\sqrt{\sigma}\|_{2}^{2} \\
& \geqslant \frac{1}{4}\|\rho-\sigma\|_{1}^{2} .
\end{aligned}
$$


In particular, $\mathrm{S}(\rho \| \sigma)=0$ if and only if $\rho=\sigma$.

Clearly, all we need to do is to rewrite a related quantity as a relative entropy with the second argument being a subnormalized state. Then, Proposition 4.1] is applied to get the desired inequality. From [3], we see that

$$
\begin{aligned}
& \mathrm{S}\left(\rho_{A B C} \| \exp \left(\log \sigma_{A B}+\log \tau_{B C}-\log \omega_{B}\right)\right) \\
& =I(A: C \mid B)_{\rho}+\mathrm{S}\left(\rho_{A B} \| \sigma_{A B}\right)+\mathrm{S}\left(\rho_{B C} \| \tau_{B C}\right)-\mathrm{S}\left(\rho_{B} \| \omega_{B}\right),
\end{aligned}
$$

where $\rho_{A B C} \in \mathrm{D}\left(\mathcal{H}_{A B C}\right), \sigma_{A C} \in \mathrm{D}\left(\mathcal{H}_{A C}\right), \tau_{B C} \in \mathrm{D}\left(\mathcal{H}_{B C}\right)$, and $\omega_{C} \in \mathrm{D}\left(\mathcal{H}_{C}\right)$. This identity leads to the following result:

$$
\begin{aligned}
& \mathrm{S}\left(\rho_{A B C} \| \exp \left(\log \sigma_{A B}+\log \sigma_{B C}-\log \sigma_{B}\right)\right) \\
& =I(A: C \mid B)_{\rho}+\mathrm{S}\left(\rho_{A B} \| \sigma_{A B}\right)+\mathrm{S}\left(\rho_{B C} \| \sigma_{B C}\right)-\mathrm{S}\left(\rho_{B} \| \sigma_{B}\right)
\end{aligned}
$$

where $\rho_{A B C}, \sigma_{A B C} \in \mathrm{D}\left(\mathcal{H}_{A B C}\right)$. Using monotonicity inequality of relative entropy, we have

$$
\mathrm{S}\left(\rho_{A B} \| \sigma_{A B}\right) \geqslant \mathrm{S}\left(\rho_{B} \| \sigma_{B}\right) \text { and } \mathrm{S}\left(\rho_{B C} \| \sigma_{B C}\right) \geqslant \mathrm{S}\left(\rho_{B} \| \sigma_{B}\right)
$$

This yields that

$$
\frac{1}{2}\left[\mathrm{~S}\left(\rho_{A B} \| \sigma_{A B}\right)+\mathrm{S}\left(\rho_{B C} \| \sigma_{B C}\right)\right] \geqslant \mathrm{S}\left(\rho_{B} \| \sigma_{B}\right) .
$$

Therefore, we obtain the following result:

Proposition 4.2. It holds that

$$
\begin{aligned}
& S\left(\rho_{A B C} \| \exp \left(\log \sigma_{A B}+\log \sigma_{B C}-\log \sigma_{B}\right)\right) \\
& \geqslant I(A: C \mid B)_{\rho}+\frac{1}{2} S\left(\rho_{A B} \| \sigma_{A B}\right)+\frac{1}{2} S\left(\rho_{B C} \| \sigma_{B C}\right),
\end{aligned}
$$

where $\rho_{A B C}, \sigma_{A B C} \in \mathrm{D}\left(\mathcal{H}_{A B C}\right)$. In particular, $\mathrm{S}\left(\rho_{A B C} \| \exp \left(\log \rho_{A B}+\log \rho_{B C}-\log \rho_{B}\right)\right) \geqslant 0$, i.e. $I(A$ : $C \mid B)_{\rho} \geqslant 0$, the strong subadditivity inequality. Moreover, $\mathrm{S}\left(\rho_{A B C} \| \exp \left(\log \sigma_{A B}+\log \sigma_{B C}-\log \sigma_{B}\right)\right)=0$ if and only if $\rho_{A B C}=\exp \left(\log \sigma_{A B}+\log \sigma_{B C}-\log \sigma_{B}\right)$.

If $S\left(\rho_{A B C} \| \exp \left(\log \sigma_{A B}+\log \sigma_{B C}-\log \sigma_{B}\right)\right)=0$, then using Proposition 4.2, we have

$$
\left\{\begin{array}{l}
I(A: C \mid B)_{\rho}=0 ; \\
S\left(\rho_{A B}|| \sigma_{A B}\right)=0 \\
S\left(\rho_{B C} \| \sigma_{B C}\right)=0
\end{array}\right.
$$

This leads to the following:

$$
\rho_{A B}=\sigma_{A B}, \rho_{B C}=\sigma_{B C} .
$$

Thus, $\rho_{B}=\sigma_{B}$, which indicates that

$$
\exp \left(\log \rho_{A B}+\log \rho_{B C}-\log \rho_{B}\right)=\exp \left(\log \sigma_{A B}+\log \sigma_{B C}-\log \sigma_{B}\right)
$$


Note that $I(A: C \mid B)_{\rho}=0$ if and only if $\exp \left(\log \rho_{A B}+\log \rho_{B C}-\log \rho_{B}\right)=\rho_{A B C}$. Therefore, $\exp \left(\log \sigma_{A B}+\right.$ $\left.\log \sigma_{B C}-\log \sigma_{B}\right)=\rho_{A B C}$. From the above-mentioned process, it follows that

$$
\mathrm{S}\left(\rho_{A B C} \| \exp \left(\log \sigma_{A B}+\log \sigma_{B C}-\log \sigma_{B}\right)\right)=0 \Longrightarrow \rho_{A B C}=\exp \left(\log \sigma_{A B}+\log \sigma_{B C}-\log \sigma_{B}\right) .
$$

We know that, for any state $\sigma_{A B C} \in \mathrm{D}\left(\mathcal{H}_{A B C}\right)$,

$$
\operatorname{Tr}\left(\exp \left(\log \sigma_{A B}+\log \sigma_{B C}-\log \sigma_{B}\right)\right) \leqslant 1
$$

But what will happen if $\operatorname{Tr}\left(\exp \left(\log \sigma_{A B}+\log \sigma_{B C}-\log \sigma_{B}\right)\right)=1$ ? In order to answer this question, we form an operator for any state $\sigma_{A B C} \in \mathrm{D}\left(\mathcal{H}_{A B C}\right)$, namely,

$$
\exp \left(\log \sigma_{A B}+\log \sigma_{B C}-\log \sigma_{B}\right)
$$

If $\exp \left(\log \sigma_{A B}+\log \sigma_{B C}-\log \sigma_{B}\right)$ is a legitimate state, denoted by $\rho_{A B C}$, then

$$
\rho_{A B}=\operatorname{Tr}_{C}\left(\exp \left(\log \sigma_{A B}+\log \sigma_{B C}-\log \sigma_{B}\right)\right), \rho_{B C}=\operatorname{Tr}_{A}\left(\exp \left(\log \sigma_{A B}+\log \sigma_{B C}-\log \sigma_{B}\right)\right),
$$

and $\rho_{B}=\operatorname{Tr}_{A C}\left(\exp \left(\log \sigma_{A B}+\log \sigma_{B C}-\log \sigma_{B}\right)\right)$. Furthermore, $S\left(\rho_{A B C} \| \exp \left(\log \sigma_{A B}+\log \sigma_{B C}-\log \sigma_{B}\right)\right)=$ 0. Thus, $I(A: C \mid B)_{\rho}=0$, i.e. $\exp \left(\log \sigma_{A B}+\log \sigma_{B C}-\log \sigma_{B}\right)$ is a Markov state.

Proposition 4.3. Given a state $\rho_{A B C}$, we form an operator $\exp \left(\log \rho_{A B}+\log \rho_{B C}-\log \rho_{B}\right)$. If

$$
\operatorname{Tr}\left(\exp \left(\log \rho_{A B}+\log \rho_{B C}-\log \rho_{B}\right)\right)=1,
$$

then the following statements are true:

(i) $\exp \left(\log \rho_{A B}+\log \rho_{B C}-\log \rho_{B}\right)=\rho_{A B}^{1 / 2} \rho_{B}^{-1 / 2} \rho_{B C} \rho_{B}^{-1 / 2} \rho_{A B}^{1 / 2}$;

(ii) $\exp \left(\log \rho_{A B}+\log \rho_{B C}-\log \rho_{B}\right)=\rho_{B C}^{1 / 2} \rho_{B}^{-1 / 2} \rho_{A B} \rho_{B}^{-1 / 2} \rho_{B C}^{1 / 2}$.

Therefore, $\exp \left(\log \rho_{A B}+\log \rho_{B C}-\log \rho_{B}\right)$ must be a Markov state.

From the above result, we see that if a state $\rho_{A B C}$ can be expressed by the form of $\exp \left(\log \sigma_{A B}+\right.$ $\left.\log \sigma_{B C}-\log \sigma_{B}\right)$ for another state $\sigma_{A B C}$, then $\rho_{A B C}$ must ba a Markov state. A question naturally arises: Which states $\rho_{A B C}$ are such that $\exp \left(\log \rho_{A B}+\log \rho_{B C}-\log \rho_{B}\right)$ is a Markov state? It would be interesting to figure out the structure of the following set:

$$
\left\{\rho_{A B C} \in \mathrm{D}\left(\mathcal{H}_{A B C}\right): \operatorname{Tr}\left(\exp \left(\log \rho_{A B}+\log \rho_{B C}-\log \rho_{B}\right)\right)=1\right\} \text {. }
$$

Theorem 4.4. For any tripartite states $\rho_{A B C}, \sigma_{A B C}, \tau_{A B C}, \omega_{A B C} \in \mathrm{D}\left(\mathcal{H}_{A B C}\right)$, if $\sigma_{B}=\tau_{B}$ or $\tau_{B}=\omega_{B}$, then

$$
\begin{aligned}
& \operatorname{S}\left(\rho_{A B C} \| \exp \left(\log \sigma_{A B}-\log \tau_{B}+\log \omega_{B C}\right)\right) \\
& \geqslant-2 \log \operatorname{Tr}\left(\sqrt{\rho_{A B C}} \sqrt{\exp \left(\log \sigma_{A B}-\log \tau_{B}+\log \omega_{B C}\right)}\right) \\
& \geqslant\left\|\sqrt{\rho_{A B C}}-\sqrt{\exp \left(\log \sigma_{A B}-\log \tau_{B}+\log \omega_{B C}\right)}\right\|_{2}^{2} \\
& \geqslant \frac{1}{4}\left\|\rho_{A B C}-\exp \left(\log \sigma_{A B}-\log \tau_{B}+\log \omega_{B C}\right)\right\|_{1}^{2} .
\end{aligned}
$$


Proof. Since $\operatorname{Tr}\left(\exp \left(\log \sigma_{A B}-\log \tau_{B}+\log \omega_{B C}\right)\right) \leqslant 1$ (see Remark 3.6), that is $\exp \left(\log \sigma_{A B}-\log \tau_{B}+\right.$ $\left.\log \omega_{B C}\right)$ is a subnormalized state, it follows from (4.1) that the desired inequality is correct.

Proposition 4.5. For a tripartite state $\rho_{A B C} \in \mathrm{D}\left(\mathcal{H}_{A B C}\right)$, it holds that

$$
\begin{aligned}
S\left(\rho_{A B}\right)+\mathrm{S}\left(\rho_{B C}\right)-\mathrm{S}\left(\rho_{A B C}\right) \geqslant & -2 \log \operatorname{Tr}\left(\sqrt{\rho_{A B C}} \sqrt{\exp \left(\log \rho_{A B}+\log \rho_{B C}\right)}\right) \\
\geqslant & \left\|\sqrt{\rho_{A B C}}-\sqrt{\exp \left(\log \rho_{A B}+\log \rho_{B C}\right)}\right\|_{2}^{2} \\
& \geqslant \frac{1}{4}\left\|\rho_{A B C}-\exp \left(\log \rho_{A B}+\log \rho_{B C}\right)\right\|_{1}^{2} .
\end{aligned}
$$

Proof. All we need to do is to rewrite $\mathrm{S}\left(\rho_{A B}\right)+\mathrm{S}\left(\rho_{B C}\right)-\mathrm{S}\left(\rho_{A B C}\right)$ as a relative entropy:

$$
\mathrm{S}\left(\rho_{A B}\right)+\mathrm{S}\left(\rho_{B C}\right)-\mathrm{S}\left(\rho_{A B C}\right)=\mathrm{S}\left(\rho_{A B C} \| \exp \left(\log \rho_{A B}+\log \rho_{B C}\right)\right)
$$

Next, we prove that $\exp \left(\log \rho_{A B}+\log \rho_{B C}\right)$ is a subnormalized state. Using Golden-Thompson inequality, we have

$$
\begin{aligned}
\operatorname{Tr}\left(\exp \left(\log \rho_{A B}+\log \rho_{B C}\right)\right) & \leqslant \operatorname{Tr}\left(\exp \left(\log \rho_{A B}\right) \exp \left(\log \rho_{B C}\right)\right) \\
& \leqslant \operatorname{Tr}\left(\rho_{A B} \rho_{B C}\right)=\operatorname{Tr}\left(\rho_{B}^{2}\right) \leqslant 1 .
\end{aligned}
$$

This completes the proof.

Further comparison with the inequalities in [12, 22] would be interesting and is left for the future research.

\section{Discussion and concluding remarks}

The lower bound in (3.18) is clearly independent of any measurement, compared with (1.8) and (1.9). Since the trace-norm decreases under generic quantum channels, in particular under partial trace, it follows that

$$
E_{s q}\left(\rho_{A C}\right) \geqslant \frac{1}{8}\left\|\rho_{A C}-\operatorname{Tr}_{B}\left(\exp \left(\log \rho_{A B}+\log \rho_{B C}-\log \rho_{B}\right)\right)\right\|_{1}^{2},
$$

where $E_{s q}$ is an entanglement measure, i.e. squashed entanglement, defined by

$$
E_{s q}\left(\rho_{A C}\right)=\inf \left\{\frac{1}{2} I(A: C \mid B)_{\rho}: \operatorname{Tr}_{B}\left(\rho_{A B C}\right)=\rho_{A C}\right\},
$$

where the infimum is taken over all possible extensions $\rho_{A B C}$ of $\rho_{A C}$. Since the squashed entanglement is a faithful measure, i.e. $E_{s q}\left(\rho_{A C}\right)=0$ if and only if $\rho_{A C}$ is a separable state, it follows that if $\rho_{A C}$ is separable, then there exists an extension $\rho_{A B C}$ of $\rho_{A C}$ such that

$$
\rho_{A C}=\operatorname{Tr}_{B}\left(\exp \left(\log \rho_{A B}+\log \rho_{B C}-\log \rho_{B}\right)\right) .
$$

Equivalently, if $\rho_{A C} \neq \operatorname{Tr}_{B}\left(\exp \left(\log \rho_{A B}+\log \rho_{B C}-\log \rho_{B}\right)\right)$ for any extension $\rho_{A B C}$ of $\rho_{A C}$, then $\rho_{A C}$ must be entangled. We would like to know wether or not

$$
\rho_{A C} \text { is separable if and only if } \rho_{A C}=\operatorname{Tr}_{B}\left(\exp \left(\log \rho_{A B}+\log \rho_{B C}-\log \rho_{B}\right)\right)
$$


for some extension $\rho_{A B C}$ of $\rho_{A C}$. From this observation, one sees that finding some properties of the following operators is a very interesting subject:

$$
\exp \left(\log \rho_{A B}+\log \rho_{B C}-\log \rho_{B}\right), \operatorname{Tr}_{B}\left(\exp \left(\log \rho_{A B}+\log \rho_{B C}-\log \rho_{B}\right)\right) \text {. }
$$

Taking the partial traces of tripartite operators maybe important in the investigation of entanglement theory. It will provide new insights in understanding entanglement.

For instance, consider the generalized Lie-Trotter product formula [24]: for any $k$ matrices $A_{1}, \ldots, A_{k}$, it holds that

$$
\lim _{n \rightarrow \infty}\left(\exp \left(A_{1} / n\right) \exp \left(A_{2} / n\right) \cdots \exp \left(A_{k} / n\right)\right)^{n}=\exp \left(A_{1}+A_{2}+\cdots+A_{k}\right) .
$$

This leads to the following identities:

$$
\begin{aligned}
\exp \left(\log \rho_{A B}-\log \rho_{B}+\log \rho_{B C}\right) & =\lim _{n \rightarrow \infty}\left(\rho_{A B}^{1 / 2 n} \rho_{B}^{-1 / 2 n} \rho_{B C}^{1 / n} \rho_{B}^{-1 / 2 n} \rho_{A B}^{1 / 2 n}\right)^{n} \\
& =\lim _{n \rightarrow \infty}\left(\rho_{B C}^{1 / 2 n} \rho_{B}^{-1 / 2 n} \rho_{A B}^{1 / n} \rho_{B}^{-1 / 2 n} \rho_{B C}^{1 / 2 n}\right)^{n}
\end{aligned}
$$

We wonder whether the sequence $\operatorname{Tr}\left(\left(\rho_{A B}^{1 / 2 n} \rho_{B}^{-1 / 2 n} \rho_{B C}^{1 / n} \rho_{B}^{-1 / 2 n} \rho_{A B}^{1 / 2 n}\right)^{n}\right)$ is monotone in $n$ and is no more than one. I proposed this conjecture in the previous version of the present paper, and luckily, Datta and Wilde [8] gave a positive answer to my question partially. In fact, they found that a key result should be cited. That is, if $M \in M_{d}(\mathbb{C})$ and $\alpha \geqslant 1$, then the map $X \mapsto \operatorname{Tr}\left(\left[M X^{1 / \alpha} M^{+}\right]^{\alpha}\right)$ is concave over Pd $\left(\mathbb{C}^{d}\right)$ [6]. In what follows, we may obtain the following:

$$
\begin{aligned}
1 & =\operatorname{Tr}\left(\left(\rho_{A B}^{1 / 2 n} \rho_{B}^{-1 / 2 n}\left[\rho_{B} \otimes \mathbb{1}_{C} / d_{C}\right]^{1 / n} \rho_{B}^{-1 / 2 n} \rho_{A B}^{1 / 2 n}\right)^{n}\right) \\
& =\operatorname{Tr}\left(\left(\rho_{A B}^{1 / 2 n} \rho_{B}^{-1 / 2 n}\left[\int_{\mathrm{U}\left(d_{C}\right)} U_{C} \rho_{B C} U_{C}^{\dagger} \mathrm{d} U_{C}\right]^{1 / n} \rho_{B}^{-1 / 2 n} \rho_{A B}^{1 / 2 n}\right)^{n}\right) \\
& \geqslant \int_{\mathrm{U}\left(d_{C}\right)} \operatorname{Tr}\left(\left(\rho_{A B}^{1 / 2 n} \rho_{B}^{-1 / 2 n}\left[U_{C} \rho_{B C} U_{C}^{\dagger}\right]^{1 / n} \rho_{B}^{-1 / 2 n} \rho_{A B}^{1 / 2 n}\right)^{n}\right) \mathrm{d} U_{C} \\
& =\operatorname{Tr}\left(\left(\rho_{A B}^{1 / 2 n} \rho_{B}^{-1 / 2 n} \rho_{B C}^{1 / n} \rho_{B}^{-1 / 2 n} \rho_{A B}^{1 / 2 n}\right)^{n}\right) .
\end{aligned}
$$

Note here that the concavity of the map $X \mapsto \operatorname{Tr}\left(\left[M X^{1 / \alpha} M^{\dagger}\right]^{\alpha}\right)$ is very essential here. The following inequality

$$
\operatorname{Tr}\left(\left(\rho_{A B}^{1 / 2 n} \rho_{B}^{-1 / 2 n} \rho_{B C}^{1 / n} \rho_{B}^{-1 / 2 n} \rho_{A B}^{1 / 2 n}\right)^{n}\right) \leqslant 1 \quad(\forall n \geqslant 1)
$$

directly leads to another proof of the fact that $\operatorname{Tr}\left(\exp \left(\log \rho_{A B}-\log \rho_{B}+\log \rho_{B C}\right)\right) \leqslant 1$ by taking the limit of Eq. (5.7) when $n \rightarrow \infty$. We can use the same technique to get a more general result: If $\rho_{A B C}, \sigma_{A B C}, \tau_{A B C} \in$ $\mathrm{D}\left(\mathcal{H}_{A B C}\right)$ and $\rho_{B}=\sigma_{B}$ or $\sigma_{B}=\tau_{B}$, then

$$
\operatorname{Tr}\left(\left(\rho_{A B}^{1 / 2 n} \sigma_{B}^{-1 / 2 n} \tau_{B C}^{1 / n} \sigma_{B}^{-1 / 2 n} \rho_{A B}^{1 / 2 n}\right)^{n}\right) \leqslant 1 \quad(\forall n \geqslant 1) .
$$

Based on this result and Stinespring's dilation representation of completely positive maps, Datta and Wilde [8, Eq. (3.50)] gave the proof of the following inequality:

$$
\operatorname{Tr}\left(\exp \left(\log \sigma+\Phi^{*}(\log \Phi(\rho))-\Phi^{*}(\log \Phi(\sigma))\right)\right) \leqslant 1 .
$$


It is natural to ask here the implication of the saturation:

$$
\operatorname{Tr}\left(\exp \left(\log \sigma+\Phi^{*}(\log \Phi(\rho))-\Phi^{*}(\log \Phi(\sigma))\right)\right)=1 .
$$

It is left open for the future research. Furthermore, we get the following improvement of monotonicity of relative entropy:

$$
\mathrm{S}(\rho \| \sigma)-\mathrm{S}(\Phi(\rho) \| \Phi(\sigma)) \geqslant \frac{1}{4}\left\|\rho-\exp \left(\log \sigma+\Phi^{*}(\log \Phi(\rho))-\Phi^{*}(\log \Phi(\sigma))\right)\right\|_{1}^{2} .
$$

Clearly the above result is not applicable in the present form since the operator

$$
\exp \left(\log \sigma+\Phi^{*}(\log \Phi(\rho))-\Phi^{*}(\log \Phi(\sigma))\right)
$$

may not be a valid state. We propose the following open questions:

$$
\begin{aligned}
\mathrm{S}(\rho \| \sigma)-\mathrm{S}(\Phi(\rho) \| \Phi(\sigma)) & \geqslant \frac{1}{4}\left\|\rho-\Phi_{\sigma}^{*} \circ \Phi(\rho)\right\|_{1}^{2}, \\
\mathrm{~S}\left(\rho_{A B} \| \sigma_{A B}\right)-\mathrm{S}\left(\rho_{A} \| \sigma_{A}\right) & \geqslant \frac{1}{4}\left\|\rho_{A B}-\sigma_{A B}^{1 / 2} \sigma_{A}^{-1 / 2} \rho_{A} \sigma_{A}^{-1 / 2} \sigma_{A B}^{1 / 2}\right\|_{1}^{2} \\
I(A: C \mid B)_{\rho} & \geqslant \frac{1}{4}\left\|\rho_{A B C}-\rho_{A B}^{1 / 2} \rho_{B}^{-1 / 2} \rho_{B C} \rho_{B}^{-1 / 2} \rho_{A B}^{1 / 2}\right\|_{1}^{2} .
\end{aligned}
$$

Apparently, (5.12) implies the remaining two inequalities. Further investigations on these topics are in order. We hope that the results obtained in our work shed new light over related subjects in quantum information theory.

\section{Acknowledgements}

The author thanks the anonymous referee for helpful comments on our manuscript, and he also thanks Uttam Singh and Zhaoqi Wu for various helpful discussions and suggestions. The work is supported by National Natural Science Foundation of China (No.11301124).

\section{References}

[1] Audenaert, K.M.R., Nussbaum, M., Szkoła, A., Verstraete, F.: Asymptotic Error Rates in Quantum Hypothesis Testing. Commun. Math. Phys. 279, 251-283 (2008)

[2] Berta, M., Lemm, M., Wilde, M.M.: Monotonicity of quantum relative entropy and recoverability. Quant. Inf. Comput. 15(15-16),1333-1354 (2015)

[3] Berta, M., Seshadreesan, K.P., Wilde, M.M.: Rényi generalizations of the conditional quantum mutual information. J. Math. Phys. 56(2), 022205 (2015)

[4] Brandão, F., Christandl, M., Yard, J.: Faithful squashed entanglement. Commun. Math. Phys. 306, 805-830 (2011) Erratum to: Faithful Squashed Entanglement, 316, 287-288 (2012) arXiv:1010.1750v5

[5] Brandão, F., Harrow, A.W., Oppenheim, Strelchuk, J.S.: Quantum conditional mutual information, reconstructed states, and state redistribution. Phys. Rev. Lett. 115, 050501 (2015) 
[6] Carlen, E.A., Lieb, E.H.: A Minkowski type trace inequality and strong subadditivity of quantum entropy II: convexity and concavity. Lett. Math. Phys. 83: 107-126 (2008)

[7] Carlen, E.A., Lieb, E.H.: Remainder terms for some quantum entropy inequalities. J. Math. Phys. 55, $042201(2014)$

[8] Datta, N., Wilde, M.M.: Quantum Markov chains, sufficiency of quantum channels, and Rényi information measures. J. Phys. A: Math. Theor. 48, 505301 (2015)

[9] Fawzi O., Renner, R.: Quantum conditional mutual information and approximate Markov chains. Commun. Math. Phys. 340(2), 575-611 (2015)

[10] Hayden, P., Jozsa, R., Petz, D., Winter, A.: Structure of states which satisfy strong subadditivity of quantum entropy with equality. Commun. Math. Phys. 246, 359-374 (2004)

[11] Hiai, F., Mosonyi, M., Petz, D., Bény, C.: Quantum f-divergences and error correction. Rev. Math. Phys. 23(7), 691-747 (2011)

[12] Kim, I.H.: Operator extension of strong subadditivity of entropy. J. Math. Phys. 53, 122204 (2012)

[13] Ibinson, B., Linden, N., Winter, A.: Robustness of Quantum Markov Chains. Commun. Math. Phys. 277, 289-304 (2008)

[14] Li, K., Winter, A.: Relative entropy and squahsed entanglement. Commun. Math. Phys. 326, 63-80 (2014)

[15] Li, K., Winter, A.: Squashed entanglement, $k$-extendibility, quantum Markov chains, and recovery maps. arXiv:1410.4184

[16] Lieb, E.H.: Convex trace functions and the Wigner-Yanase-Dyson conjecture. Adv. Math. 11, 267-288 (1973)

[17] Lieb, E.H., Ruskai, M.: Proof of the strong subadditivity of quantum-mechanical entropy. J. Math. Phys. 14, 1938-1941 (1973)

[18] Mosonyi, M., Hiai, F.: On the Quantum Rényi Relative Entropies and Related Capacity Formulas. IEEE Trans. Inf. Theor. 57, 2474-2487 (2011)

[19] Petz, D.: Sufficiency of channels over von Neumann algebras. Quart. J. Math. 39 (1), 97-108 (1988)

[20] Petz, D.: Monotonicity of quantum relative entropy revisited. Rev. Math. Phys. 15, 79-91 (2003)

[21] Ruskai, M.B.: Inequalities for quantum entropy: A review with conditions for equality. J. Math. Phys. 43, 4358-4375 (2002): Erratum 46, 019901 (2005)

[22] Ruskai, M.B.: Remarks on on Kim's strong subadditivity matrix inequality: extensions and equality conditions. J. Math. Phys. 54, 102202 (2013)

[23] Seshadreesan, K.P. , Berta, M., Wilde, M.M.: Rényi squashed entanglement, discord, and relative entropy differences. J. Phys. A: Math. Theor. 48, 395303 (2015) 
[24] Suzuki, M.: Transfer-matrix method and Monte Carlo simulation in quantum spin systems. Phys. Rev. B 31, 2957 (1985)

[25] Zhang, L.: Conditional mutual information and commutator. Int. J. Theor. Phys. 52(6): 2112-2117 (2013)

[26] Zhang, L.: Matrix integrals over unitary groups: An application of Schur-Weyl duality. arXiv:1408.3782

[27] Zhang, L., Wu, J.: A lower bound of quantum conditional mutual information. J. Phys. A: Math. Theor. 47, 415303 (2014) 